\title{
ARTICLE
}

\section{The horizontal reconstructive ladder}

W G Kleintjes, MMed (Plast), FC (Plast Surg) SA, PhD

Western Cape Provincial Tertiary Adult Burn Unit, Division of Surgery, Tygerberg Hospital and Stellenbosch University, South Africa

Corresponding author: W G Kleintjes (waynekleintjes@yahoo.com)

\begin{abstract}
The reconstructive ladder has been a traditional teaching model for the selection of reconstructive surgery options for many years. Some publications in recent decades have proposed that the reconstructive ladder is no longer applicable, because many new adjunctive reconstructive options are difficult to incorporate into it. An alternative method of thinking about the integration of these adjunctive techniques in the reconstructive ladder is the horizontal reconstructive ladder. This ladder, as an adjunct to the traditional vertical reconstructive ladder, has the potential and vertical adaptability to assist in teaching the reconstructive arsenal of the plastic surgeon.
\end{abstract}

SAfr J Plast Reconstr Aesthet Surg Burns 2018;1 (1):19-20. DOI: 10.7196/SAJPRASB.2018.v1i1.7

The reconstructive ladder has been a traditional teaching model for the selection of reconstructive surgery options for many years. Some publications in recent decades have proposed that the reconstructive ladder is no longer applicable, because many new adjunctive reconstructive options are difficult to incorporate into it. ${ }^{[1,2]}$

Some authors have suggested adding additional or adjunctive procedures into the vertical ladder. ${ }^{[3]}$

This did not make good sense, and some have proposed that the vertical ladder be abandoned. ${ }^{[1,2]} \mathrm{A}$ reconstructive elevator model was proposed as a better way of selecting reconstructive options. ${ }^{[2]}$ Several other methods have also been previously proposed. ${ }^{[4,5]}$

\section{Proposed concept}

An alternative way of thinking about the integration of adjunctive techniques with the reconstructive ladder is their incorporation into a horizontal reconstructive ladder.

Several adjuncts that assist with wound closure have been developed in recent years, such as negative pressure wound therapy, wound therapies such as hyperbaric oxygen, tissue expansion, osteodistraction, lipofilling and epidermal and dermal skin substitutes. These adjuncts were not part of the original vertical reconstructive ladder.

This horizontal ladder can be applied at any level of the vertical reconstructive ladder, as was previously proposed in the reconstructive elevator concept. ${ }^{[2]}$

The combined horizontal and vertical reconstructive ladder is dynamic and versatile, and is shown in Fig. 1.

\section{Discussion}

The reconstructive ladder, over the years, has had to adapt to changes in surgical options as they were developed and found a place in the skill set of the reconstructive surgeon. ${ }^{[4-6]}$ Some have suggested that reconstruction options be considered in stages, as an alternative to the reconstructive ladder, but this has not been accepted by all. ${ }^{[7]}$

Adding adjunctive treatments directly onto the vertical ladder, where their position would need to be fixed, would create problems for the reconstructive

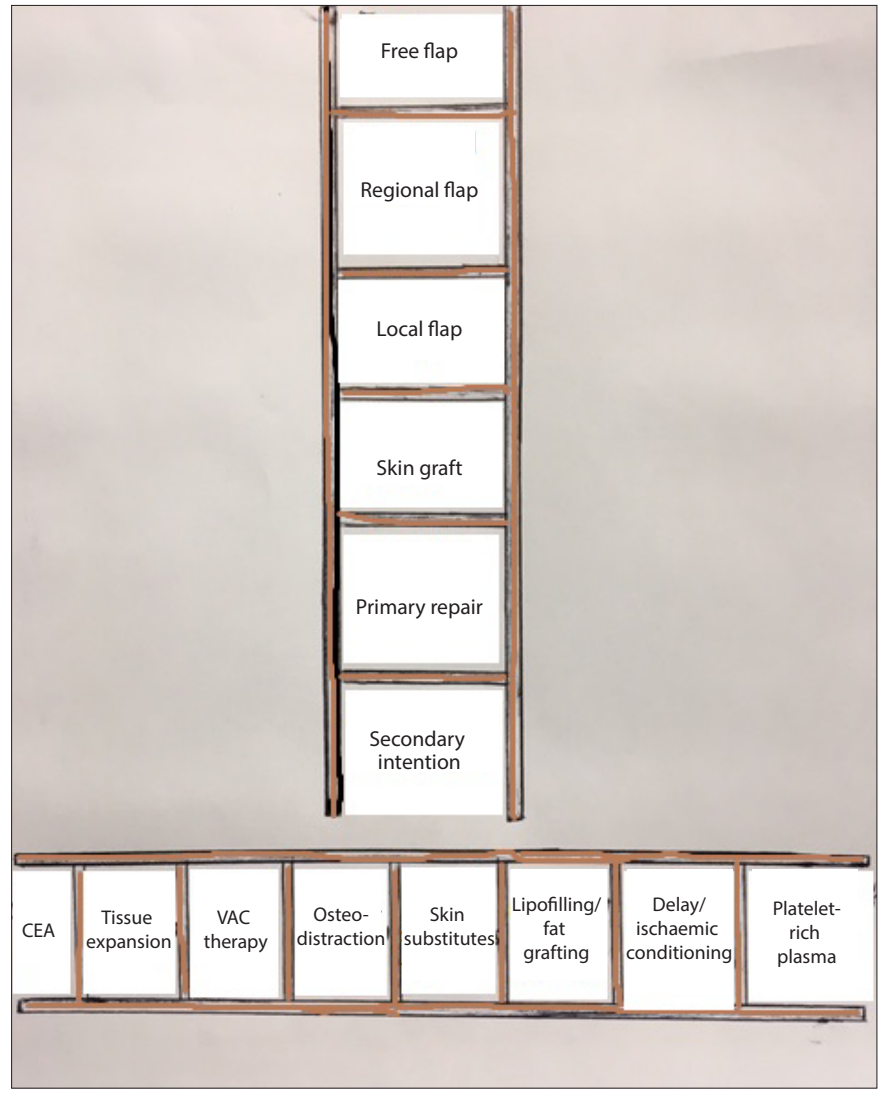

Fig. 1. The horizontal reconstructive ladder in relation to the vertical reconstructive ladder. The horizontal options are adjuncts to surgery, and can be applied at any level of the vertical ladder. Not all adjunctive options are included on the horizontal ladder. The horizontal options usually decrease the demand for higher options on the vertical ladder. (CEA = cultured epithelial autografts; VAC = vacuum-assisted closure).

surgeon. It would take away the flexibility (plasticity) in reconstructive option selection that reconstructive surgeons require in practice. The versatility of these adjuncts is therefore best illustrated by a horizontal ladder that can be 
adjusted to any vertical level. Practically, the use of a horizontal option should lead to fewer requirements on the vertical ladder. For example, if negative pressure is applied to a large open wound, where traditionally one would require a flap to cover the defect, a simple skin graft may be adequate after the wound bed has granulated. The horizontal options thus assist by decreasing the demands on the vertical ladder.

The vertical ladder is still a useful way to think about the ease or complexity of closing defects. Donor sites have different morbidities, and a skin graft may still be easier to use than a local flap. Ultimately, however, donor site and ease of surgery are not the only determinants in choice of reconstruction. For example, when reconstructing a nose after a tumour excision, a skin graft may give a suboptimal cosmetic result, with possibly less donor site morbidity than a local flap of good quality and texture. Multiple variables may thus affect the best choice of reconstructive option for a patient.

The ladders should not be used as absolute rules, but rather as guidelines for reconstructive options. The vertical options reflect an increase in complexity. The horizontal options could assist in decreasing the complexity of the vertical requirement, by decreasing the vertical level. At the core of great and successful reconstructions lies the ability of the reconstructive surgeon to select the best option for the individual patient, holistically. In this regard, Sandberg proposed a plastic surgery compass, to consider factors such as risk (south), anatomical problems (west), procedural complexity (north) and personal factors (east). ${ }^{[8]}$

With many new advances in wound care, one would expect that new adjuncts to assist with wound care and reconstructive options will develop. These can be added to the ladders where they best fit.

\section{Conclusion}

The horizontal reconstructive ladder as an adjunct to the traditional vertical reconstructive ladder has the potential, and vertical plasticity, to assist in teaching, and toaugment the reconstructive arsenal of the surgeon. It is simple and easy to teach.

\section{Acknowledgements. None.}

Author contributions. Sole author.

\section{Funding. None.}

\section{Conflicts of interest. None.}

. Al Deek NF, Wei FC. It is the time to say good bye to the reconstructive ladder/lift and its variants. Plas Reconstr Aesthet Surg 2017;70(4):539-540. https://doi.org/10.1016/j.bjps.2016.12.018

Gottlieb L, Krieger LM. From the reconstructive ladder to the reconstructive elevator. Plast Reconstr Surg 1994;93(7):1503-154

3. Janis JE, Kwon RK, Attinger CE. The new reconstructive ladder: Modifications to the traditional model. Plast Reconstr Surg 2011:127(Suppl 1):S205-S212. https://doi.org/10.1097/PRS.0b013e318201271c

4. Ullmann $Y$, Fodor $L$, Ramon $Y$, et al. The revised 'reconstructive ladder' and its application for highenergy injuries to the extremities. Ann Plast Surg 2006; 56(4):401-405.

5. Knobloch K, Vogt PM. The reconstructive clockwork of the twenty-first century: An extension of the concept of the reconstructive ladder and reconstructive elevator. Plast Reconstr Surg 2010:126(4):220e-222e. https://doi.org/10.1097/PRS.0b013e3181ecleef

6 Knobloch K Vogt PM. The reconstructive ladder in light of evidence-based medicine. Plast Reconst Surg 2011;127(2):1017-1018. https://doi.org/10.1097/PRS.0b013e318200ad2b

7. Wong $\mathrm{CJ}$, Niranjan $\mathrm{N}$. Reconstructive stages as an alternative to the reconstructive ladder. Plast Reconstr Surg 2008;121(5): 362e-363e. https://doi.org/10.1097/PRS.0b013e31816b1171

8. Sandberg LJM. The plastic surgery compass:Navigating the reconstructive ladder in the personalised healthcare era. Plast Reconstr Surg Glob Open 2016;4(9):e1035. https://doi.org/10.1097/ GOX.0000000000001035

Accepted 8 November 2018 